\title{
Higher liver stiffness in patients with chronic congestive heart failure: data from NHANES with liver ultrasound transient elastography
}

\author{
Meiying Zeng ${ }^{1 *}$, Yu Chen ${ }^{2 \#}$, Binghui Zhao ${ }^{1}$ \\ ${ }^{1}$ Department of Radiology, Shanghai Tenth People's Hospital, Tongji University School of Medicine, Shanghai, China; ${ }^{2}$ Department of Cardiology, \\ Shanghai Jiao Tong University Affiliated Sixth People's Hospital, Shanghai, China \\ Contributions: (I) Conception and design: B Zhao, Chen Y; (II) Administrative support: B Zhao; (III) Provision of study materials or patients: M Zeng; \\ (IV) Collection and assembly of data: M Zeng; (V) Data analysis and interpretation: M Zeng, Y Chen; (VI) Manuscript writing: All authors; (VII) \\ Final approval of manuscript: All authors. \\ \#The authors contributed equally to the work. \\ Correspondence to: Binghui Zhao, MD. Department of Radiology, Shanghai Tenth People's Hospital, Tongji University School of Medicine, Shanghai \\ 200072, China. Email: binghuizhao@163.com.
}

\begin{abstract}
Backgrounda Liver stiffness in patients with chronic congestive heart failure (CCHF) is poorly understood and liver ultrasound transient elastography (LUTE) is a new non-invasive method to detect this condition. In this cross-sectional study we explored liver stiffness and secondary congestive hepatopathy in patients with CCHF detected by LUTE.

Methods: Data from the National Health and Nutrition Examination Survey (NHANES 2017-2018) were analyzed. All participants undergoing LUTE and without liver disease were included, among whom, 110 participants were diagnosed with CCHF. The cut-off values of stiffness for fibrosis and cirrhosis were above 7.65 and $13.01 \mathrm{kPa}$, respectively. Data regarding liver stiffness were compared between the participants with and without CCHF.
\end{abstract}

Results: Among patients with CCHF, the median liver stiffness was $6.0 \mathrm{kPa}$, above $7.65 \mathrm{kPa}$ in $32.7 \%$ of patients, and above $13.01 \mathrm{kPa}$ in $14.6 \%$ of patients. The mean liver stiffness was $5.0 \mathrm{kPa}$ in the control group and was significantly lower than that of patients with $\mathrm{CCHF}(\mathrm{P}<0.001)$. The ratio of serum albumin/globulin (A/G) gradually decreased according to the liver stiffness of patients with $\mathrm{CCHF}(\mathrm{P}=0.03)$.

Conclusions: Patients with CCHF had higher liver stiffness values than controls, nearly one-third had substantial fibrosis, and more than one in seven patients progressed to cirrhosis. The A/G ratio may be a potential biomarker for liver stiffness caused by CCHF.

Keywords: Chronic congestive heart failure (CCHF); liver stiffness; liver ultrasound transient elastography (LUTE)

Submitted May 07, 2021. Accepted for publication Jun 07, 2021.

doi: 10.21037/apm-21-1362

View this article at: https://dx.doi.org/10.21037/apm-21-1362

\section{Introduction}

Chronic congestive heart failure (CCHF) is a serious condition, the prevalence of which is increasing worldwide, particularly among those $>60$ years of age (1-3). CCHF occurs in the final stages of many heart conditions and can cause elevated pressure in the right atrium and ventricle leading to liver congestion. In turn, this can stimulate connective tissue hyperplasia, cause liver fibrosis and other liver organic lesions, and even cause cirrhosis of the liver (4-6).

Hepatic fibrosis is typically seen in the early stages of congestive liver disease, and while liver biopsy is the 
traditional gold standard diagnostic method, its invasive nature limits its use (7-9). Liver ultrasound transient elastography (LUTE) is a non-invasive method which has been widely used to detect liver fibrosis (10-12) and in recent years, several studies have assessed liver fibrosis in patients with acute decompensated heart failure (ADHF) using this method. The results of these investigations has shown that liver stiffness at admission is related to clinical congestion and volume overload, that the median values of liver stiffness decreases during hospitalization, and that liver stiffness at discharge is associated with all-cause death and HF readmission (13-17). However, these studies have only discussed liver fibrosis seen in acute aggravated heart failure during hospitalization, and research on liver fibrosis in patients with CCHF after discharge is rare.

The National Health and Nutrition Examination Survey (NHANES) is a programme of studies designed to assess the health and nutritional status of adults and children in the United States that combines the interviews and physical examinations of thousands of households, which do not include ADHF or hospitalized patients (18). In this study, we performed a cross-sectional study to explore liver stiffness in patients with and without CCHF using LUTE, by analyzing data from NHANES 2017-2018. We present the following article in accordance with the STROBE reporting checklist (available at https://dx.doi. org/10.21037/apm-21-1362).

\section{Methods}

\section{Study population and data collection}

In the cross-sectional study, participants were drawn from the NHANES (2017-2018) released in Feb 2020. All NHANES participants provided informed consent. All participants who underwent LUTE were included and those with liver disease were excluded from our study. Liver stiffness and baseline data, such as demographic data, physical examination, laboratory data, and questionnaire data, were collected and compared between participants with and without CCHF, the latter who were designated as a control group. All procedures performed in this study involving human participants were in accordance with the Declaration of Helsinki (as revised in 2013). The study was waived from review by the Institutional Review Board of Shanghai Tenth People's Hospital, Tongji University School of Medicine.

\section{LUTE measurement}

In the NHANES, LUTE was performed in Mobile Examination Centers (MEC) using a FibroScan ${ }^{\circledR}$ model 502 V2 Touch with a medium (M) or extra-large (XL) wand (probe), where liver stiffness was measured and recorded as kilopascals. A detailed description of the procedures is documented in the LUTE procedures manual of NHANES (19). The predefined stiffness cut-off values were greater than $7.65 \mathrm{kPa}$ for substantial fibrosis and greater than $13.01 \mathrm{kPa}$ for cirrhosis (20).

\section{Statistical analysis}

Continuous data were presented as mean $\pm \mathrm{SD}$ or median ( $25 \%$ percentile, $75 \%$ percentile) according to whether the data were normally distributed, and categorical variables were presented as percentages. Comparisons among groups were performed using Student's $t$-test or one-way analysis of variance for variables of normal distribution and nonparametric tests for variables of non-normal distribution. $\mathrm{P}<0.05$ was statistically significant.

\section{Results}

\section{General information of the study}

A total of 5,950 participants underwent LUTE in NHANES (2017-2018), and after excluding 1,361 patients with liver disease, 4,589 participants were included in the study, among whom 110 had CCHF (Figure 1). The median age of the CCHF group was 69 years (61-77 years), and $68.2 \%$ of cases were male (Table 1). Compared with the control group, the CCHF group had considerably higher rates of hypertension (HBP), diabetes (DM), obesity, coronary heart disease (CAD), and chronic obstructive pulmonary disease (COPD), all of which are risk factors or direct causes of CCHF.

\section{Baseline characteristics of included participants}

The median stiffness of the CCHF group was $6.0 \mathrm{kPa}$, which was higher than that of the control $[6.0(4.5-10.0)$ vs. 5.0 (4.0-6.1) $\mathrm{kPa}, \mathrm{P}<0.0001]$. ALP and GGT were significantly higher in the CCHF group than in the control group $(\mathrm{P}<0.001$ and $\mathrm{P}=0.035$, respectively), and total bilirubin was mildly elevated in the CCHF group compared to the control group (8.31 vs. $7.82, \mathrm{P}=0.37$ ). 


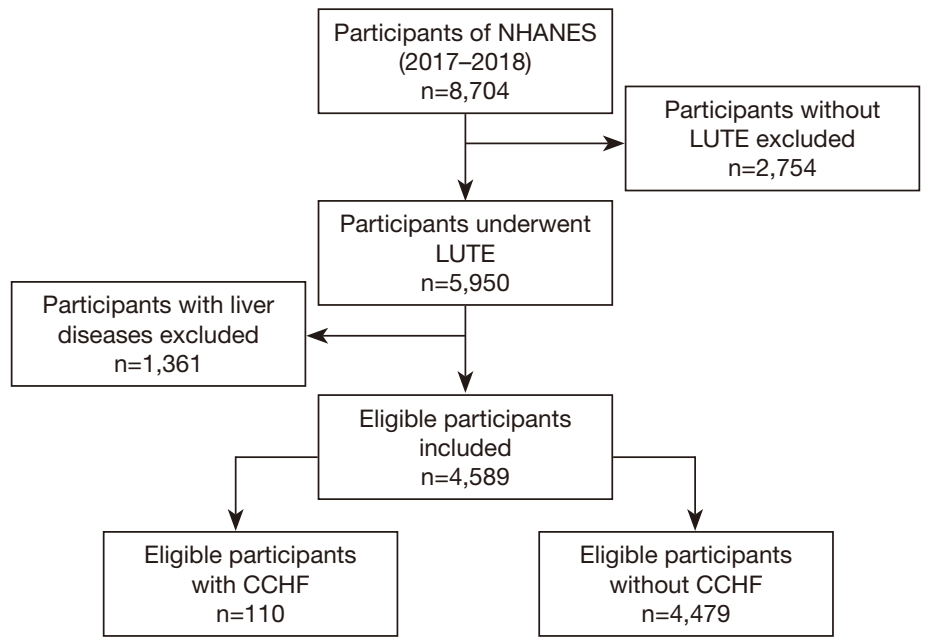

Figure 1 Flow chart of participant selection. NHANES, National Health and Nutrition Examination Survey; LUTE, liver ultrasound transient elastography; CCHF, chronic congestive heart failure.

Albumin was significantly lower in the CCHF group than in the control group $(\mathrm{P}<0.001)$, while globulin was slightly higher $(\mathrm{P}=0.08)$. The platelet count was significantly lower in the CCHF group than in the control group (219.2 vs. 244.6, $\mathrm{P}<0.001$ ), and all renal function indexes, such as creatinine, urea nitrogen, and uric acid, were significantly greater in the CCHF group than in the control group $(\mathrm{P}<0.001)$. Glycohemoglobin and fasting blood glucose were significantly higher in the CCHF group than in the control group $(\mathrm{P}<0.001)$, and $\mathrm{CRP}$ was significantly higher in the CCHF group than in the control group (8.94 vs. $3.88, \mathrm{P}=0.01)$. Finally, $\mathrm{LDH}$ was significantly higher in the CCHF group than in the control group (175.1 vs. 158.2, $\mathrm{P}=0.019)$, and the serum levels of hepatic transaminases (ALT and AST) were similar between the two groups.

\section{CCHF patients stratified by liver stiffness value}

Patients with CCHF were further stratified into a low stiffness (LS) group (74/110, 67.27\%), fibrosis group (20/110, 18.18\%), and cirrhosis group (16/110, 14.55\%) according to the cut-off values of fibrosis and cirrhosis (19) (Table 2). While hepatic aminotransferase levels, including AST and ALT, gradually increased according to liver stiffness, they did not reach statistical significance. The serum globulin level gradually also increased $(\mathrm{P}=0.01)$, while the serum albumin level, the ratio of albumin/globulin (A/G), and the platelet counts gradually decreased according to liver stiffness $(\mathrm{P}=0.26, \mathrm{P}=0.03$ and $\mathrm{P}=0.011$, respectively).

\section{Discussion}

NHANES is a nationwide survey assessing the health and nutritional status of large segments of the U.S population (18). As the participants were not hospitalized, the findings likely reflect the reality of the conditions surveyed, including CCHF, in the general population. Our results showed $2.4 \%$ of the surveyed population had CCHF, which is a figure similar to recent heart disease and stroke statistics of the American Heart Association (21). We found the liver stiffness of patients with CCHF was significantly higher than that of the control group and the median stiffness of CCHF patients was $6.0(4.5-10.0) \mathrm{kPa}$. Further, most patients $(67.27 \%)$ had no or mild liver stiffness, while a few patients progressed to liver fibrosis $(18.18 \%)$ and cirrhosis (14.55\%). These findings differ from the results of studies investigating patients with acute heart failure. In 2010, Colli explored 27 hospitalized patients with $\mathrm{ADHF}(15)$ and found the median admission and discharge stiffness values were $8.80 \mathrm{kPa}(5.92-11.90 \mathrm{kPa})$ and $7.20 \mathrm{kPa}$ $(5.20-11.30 \mathrm{kPa})$, respectively, while at admission, $14(58 \%)$ patients had liver fibrosis, five of whom $(21 \%)$ had cirrhosis. In 2013, Alegre explored 10 hospitalized patients with $\mathrm{ADHF}$ and found that the median admission and discharge stiffness values were $14.4 \mathrm{kPa}(8.3-18.8 \mathrm{kPa})$ and $8.2 \mathrm{kPa}$ (5.1-11.2 kPa), respectively (17). In another study, Soloveva explored 149 hospitalized patients with ADHF and found $85.9 \%$ had significant stiffness (13). The median values of liver stiffness were $12.2(6.3-23.6) \mathrm{kPa}$ on admission and $8.7(5.9-14.4) \mathrm{kPa}$ at discharge. In addition, Saito explored 
Table 1 Baseline characteristics of included participants

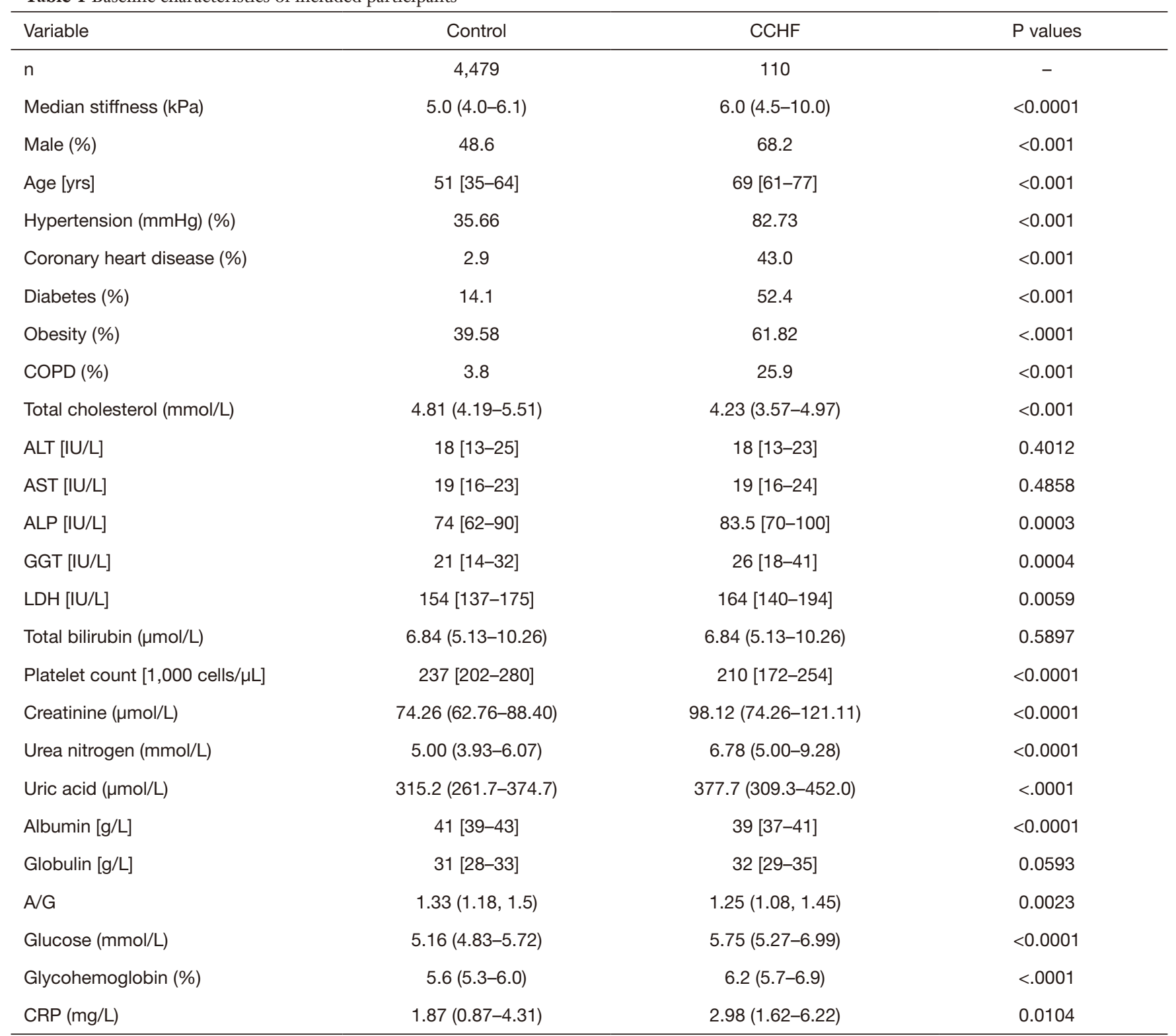

CCHF, chronic congestive heart failure; COPD, chronic obstructive pulmonary disease, ALP, alkaline phosphatase; ALT, alanine transaminase; AST, aspartate aminotransferase; GGT, $\gamma$-glutamyl transpeptidase; LDH, lactate dehydrogenase; A/G, albumin/globulin; CRP, C-reactive protein.

105 hospitalized patients with ADHF and found the median liver stiffness to be 8.8 (4.9-21.6) $\mathrm{kPa}$, and $50.5 \%$ had high stiffness (14). Patients with ADHF in the above studies had higher proportions of increased liver stiffness and median liver stiffness values than patients with CCHF in our study, suggesting that less liver stiffness is more likely to be caused by $\mathrm{CCHF}$ in comparison to $\mathrm{ADHF}$.
CCHF may cause liver congestion and stiffness, subsequent fibrosis, and cirrhosis, which is referred to as congestive hepatopathy (22-25). Transaminases are important biomarkers of hepatic injury and are elevated in severe liver fibrosis and cirrhosis (26,27). However, Allen found that ALT and AST were less commonly abnormal in 2,679 patients with CCHF when analyzing results from the 
Table 2 Laboratory data of CCHF patients stratified by liver stiffness value

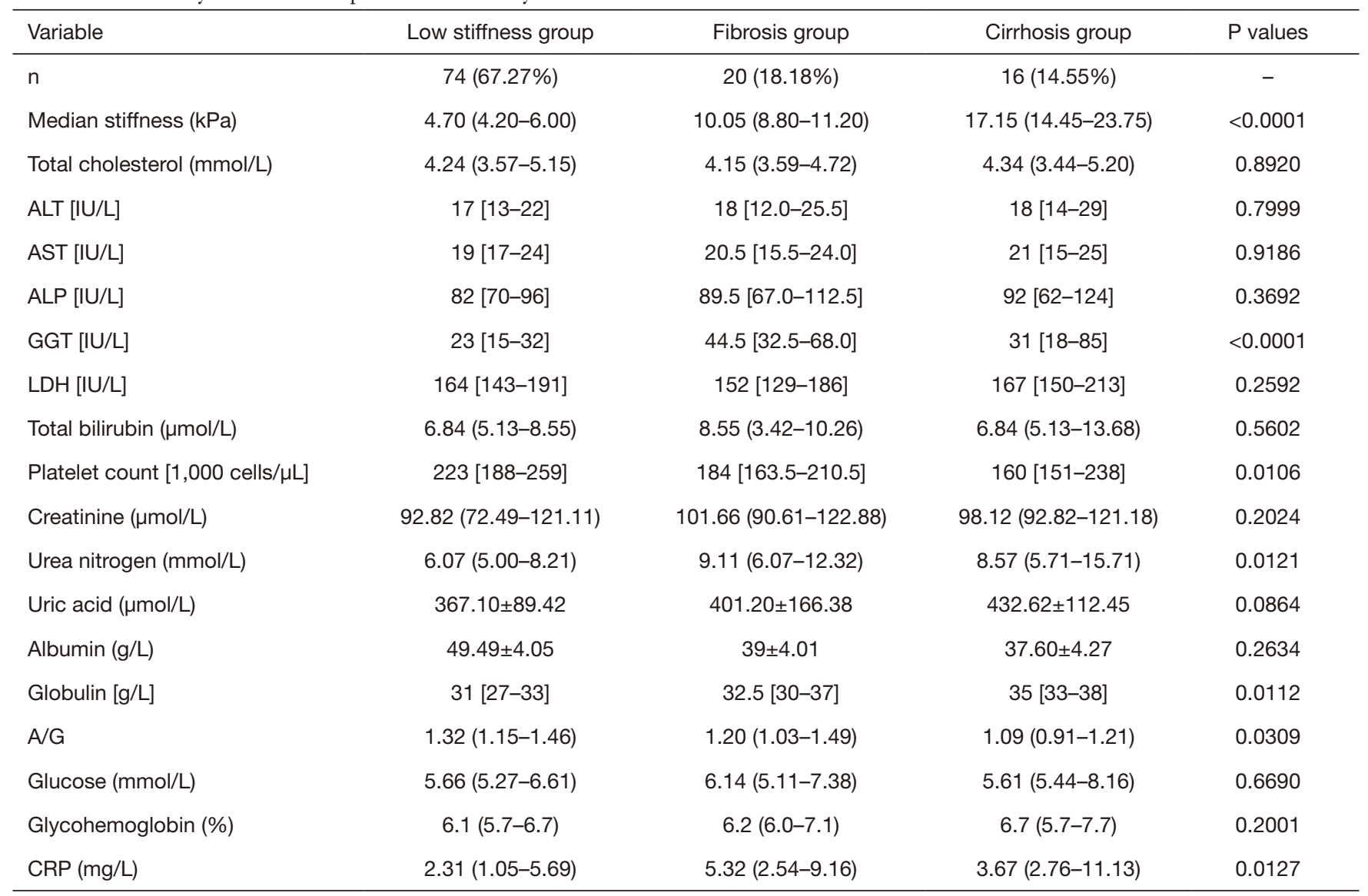

CCHF, chronic congestive heart failure; ALP, alkaline phosphatase; ALT, alanine transaminase; AST, aspartate aminotransferase; GGT, $\gamma$-glutamyl transpeptidase; LDH, lactate dehydrogenase; A/G, Albumin/Globulin; CRP, C-reactive protein.

CHARM study (28), which is consistent with our results and those of Soloveva and Saito $(13,14)$. This implies most hepatic injuries caused by heart failure are in the compensatory period and are not serious.

Cholestasis is characterized by rising serum bilirubin, ALP, and GGT levels. In Soloveva study, ADHF patients with moderate and high liver stiffness had higher serum bilirubin levels than those without (13). In addition, in Saito's study, ADHF patients with high stiffness presented with higher serum bilirubin and GGT levels (14). In our study, ALP and GGT increased significantly in CCHF patients compared to controls, while the total bilirubin level did not differ significantly between the two. However, none of these parameters varied significantly from the low stiffness group to the high stiffness group.

Serious liver fibrosis causes liver dysfunction, and especially affects the synthetic function of cholesterol and protein $(29,30)$. In Saito's study, patients with ADHF with high liver stiffness presented with lower total cholesterol (TC), while albumin did not change significantly (14). In our study, both TC and albumin decreased significantly in CCHF patients compared to controls, while the $\mathrm{A} / \mathrm{G}$ ratio decreased significantly. Furthermore, compared to CCHF with low stiffness, the $A / G$ of patients with moderate or high stiffness decreased significantly, although TC did not change significantly in patients with moderate or high stiffness. Our findings suggest hepatic congestion caused by $\mathrm{CCHF}$ has a greater effect on the synthesis of protein than cholesterol, which differs to that seen in ADHF.

Thrombocytopenia is a common finding in cirrhosis and is mainly caused by hypersplenism caused by portal HBP $(31,32)$. Saito et al. (14) found ADHF patients with high liver stiffness presented with lower platelet counts, and in our study, platelet levels in patients with CCHFs decreased 
significantly compared to the control. Furthermore, compared to CCHF patients with low stiffness, the platelet counts of patients with moderate or high stiffness decreased significantly, which may imply that hypersplenism is common in ADHF- and CCHF-induced congestive hepatopathy.

CRP is an important inflammatory factor (33) and recent studies have shown it is associated with liver fibrosis and cirrhosis (34-36). In our study, CRP increased significantly in the CCHF group compared to controls. Furthermore, compared to CCHF patients with low stiffness, the CRP levels of patients with moderate or high stiffness increased significantly, suggesting CRP is positively associated with liver fibrosis caused by CCHF and is sensitive to liver stiffness grades.

There are two limitations to the current study. First, as there is a lack of heart function indexes, such as BNP and echocardiographic parameters, we could not explore the relationship between heart function and the grade of liver fibrosis. Second, as a cross-sectional study, NHANES does not provide data about clinical outcomes, which prevented us from investigating the relationship between the liver stiffness of patients with CCHF and prognosis. Future prospective studies investigating cardiac function and clinical outcomes are warranted.

\section{Conclusions}

Patients with CCHF have higher liver stiffness values than patients without. Nearly early one-third of CCHF patients have substantial fibrosis, and more than one in seven patients progress to cirrhosis, which is less than that of patients with $\mathrm{ADHF}$. The $\mathrm{A} / \mathrm{G}$ ratio decreased gradually with increasing stiffness and may be a potential biomarker for liver stiffness caused by CCHF.

\section{Acknowledgments}

The authors would like to sincerely thank Dr. Ye JiangFeng from the Division of Obstetrics and Gynaecology, KK Women's and Children's Hospital for assistance with the statistical analysis.

Funding: The study was funded by the National Natural Science Foundation of China (No. 81603327). The funder had no role in study design, data collection and analysis, decision to publish, or preparation of the manuscript.

\section{Footnote}

Reporting Checklist: The authors have completed the STROBE reporting checklist. Available at https://dx.doi. org/10.21037/apm-21-1362

Conflicts of Interest: All authors have completed the ICMJE uniform disclosure form (available at https://dx.doi. org/10.21037/apm-21-1362). The authors have no conflicts of interest to declare.

Ethical Statement: The authors are accountable for all aspects of the work in ensuring that questions related to the accuracy or integrity of any part of the work are appropriately investigated and resolved. The study was waived from review by the Institutional Review Board of Shanghai Tenth People's Hospital, Tongji University School of Medicine. Participants were drawn from the NHANES (2017-2018) released in Feb 2020. All NHANES participants provided informed consent. All procedures performed in this study involving human participants were in accordance with the Declaration of Helsinki (as revised in 2013).

Open Access Statement: This is an Open Access article distributed in accordance with the Creative Commons Attribution-NonCommercial-NoDerivs 4.0 International License (CC BY-NC-ND 4.0), which permits the noncommercial replication and distribution of the article with the strict proviso that no changes or edits are made and the original work is properly cited (including links to both the formal publication through the relevant DOI and the license). See: https://creativecommons.org/licenses/by-nc-nd/4.0/.

\section{References}

1. Ziaeian B, Fonarow GC, et al. Epidemiology and aetiology of heart failure. Nat Rev Cardiol 2016;13:368-78.

2. Triposkiadis F, Xanthopoulos A, Parissis J, et al. Pathogenesis of chronic heart failure: cardiovascular aging, risk factors, comorbidities, and disease modifiers. Heart Fail Rev 2020. [Epub ahead of print] doi:10.1007/s10741020-09987-z.

3. National Guideline C. National Institute for Health and Care Excellence: Clinical Guidelines. Chronic Heart Failure in Adults: Diagnosis and Management. London: 
National Institute for Health and Care Excellence (UK) Copyright (C) NICE 2018; 2018.

4. Xanthopoulos A, Starling RC, Kitai T, et al. Heart Failure and Liver Disease: Cardiohepatic Interactions. JACC Heart Fail 2019;7:87-97.

5. Giallourakis CC, Rosenberg PM, Friedman LS, et al. The liver in heart failure. Clin Liver Dis 2002;6:947-67, viii-ix.

6. Sundaram V, Fang JC, et al. Gastrointestinal and Liver Issues in Heart Failure. Circulation 2016;133:1696-703.

7. Papastergiou V, Tsochatzis E, Burroughs AK, et al. Noninvasive assessment of liver fibrosis. Ann Gastroenterol 2012;25:218-31.

8. Saleh HA, Abu-Rashed AH, et al. Liver biopsy remains the gold standard for evaluation of chronic hepatitis and fibrosis. J Gastrointestin Liver Dis 2007;16:425-6.

9. Sharma S, Khalili K, Nguyen GC, et al. Non-invasive diagnosis of advanced fibrosis and cirrhosis. World J Gastroenterol 2014;20:16820-30.

10. Afdhal NH. Fibroscan (transient elastography) for the measurement of liver fibrosis. Gastroenterol Hepatol (N Y) 2012;8:605-7.

11. Sigrist RMS, Liau J, Kaffas AE, et al. Ultrasound Elastography: Review of Techniques and Clinical Applications. Theranostics 2017;7:1303-29.

12. Shen YN, Zheng ML, Guo CX, et al. The role of imaging in prediction of post-hepatectomy liver failure. Clin Imaging 2018;52:137-45.

13. Soloveva A, Kobalava Z, Fudim M, et al. Relationship of Liver Stiffness With Congestion in Patients Presenting With Acute Decompensated Heart Failure. J Card Fail 2019;25:176-87.

14. Saito Y, Kato M, Nagashima K, et al. Prognostic Relevance of Liver Stiffness Assessed by Transient Elastography in Patients With Acute Decompensated Heart Failure. Circ J 2018;82:1822-9.

15. Colli A, Pozzoni P, Berzuini A, et al. Decompensated chronic heart failure: increased liver stiffness measured by means of transient elastography. Radiology 2010;257:872-8.

16. Taniguchi T, Ohtani T, Kioka H, et al. Liver Stiffness Reflecting Right-Sided Filling Pressure Can Predict Adverse Outcomes in Patients With Heart Failure. JACC Cardiovasc Imaging 2019;12:955-64.

17. Alegre F, Herrero JI, Iñarrairaegui M, et al. Increased liver stiffness values in patients with heart failure. Acta Gastroenterol Belg 2013;76:246-50.

18. Center for Disease Control and Prevention NCfHS. National Health and Nutrition Examination Survey. Available online: http://www.cdc.gov/nchs/nhanes/about_ nhanes.htm. (Accessed September 27, 2020).

19. Statistics CNCfH. NHANES 2017-2018 Procedure

Manuals, Available online: https://wwwn.cdc. gov/nchs/nhanes/ContinuousNhanes/Manuals. aspx? BeginYear=2017; 2020.

20. Friedrich-Rust M, Ong MF, Martens S, et al. Performance of transient elastography for the staging of liver fibrosis: a meta-analysis. Gastroenterology 2008;134:960-74.

21. Benjamin EJ, Muntner P, Alonso A, et al. Heart Disease and Stroke Statistics-2019 Update: A Report From the American Heart Association. Circulation 2019;139:e56-e528.

22. Giallourakis CC. Liver complications in patients with congestive heart failure. Gastroenterol Hepatol (N Y) 2013;9:244-6.

23. Lemmer A, VanWagner L, Ganger D, et al. Congestive hepatopathy: Differentiating congestion from fibrosis. Clin Liver Dis (Hoboken) 2017;10:139-43.

24. Ostovaneh MR, Ambale-Venkatesh B, Fuji T, et al. Association of Liver Fibrosis With Cardiovascular Diseases in the General Population: The Multi-Ethnic Study of Atherosclerosis (MESA). Circ Cardiovasc Imaging 2018;11:e007241.

25. Nallagangula KS, Nagaraj SK, Venkataswamy L, et al. Liver fibrosis: a compilation on the biomarkers status and their significance during disease progression. Future Sci OA 2018;4:FSO250.

26. McGill MR. The past and present of serum aminotransferases and the future of liver injury biomarkers. EXCLI J 2016;15:817-28.

27. Cequera A, García de León Méndez MC, et al. Biomarkers for liver fibrosis: advances, advantages and disadvantages. Rev Gastroenterol Mex 2014;79:187-99.

28. Allen LA, Felker GM, Pocock S, et al. Liver function abnormalities and outcome in patients with chronic heart failure: data from the Candesartan in Heart Failure: Assessment of Reduction in Mortality and Morbidity (CHARM) program. Eur J Heart Fail 2009;11:170-7.

29. Parola M, Pinzani M, et al. Liver fibrosis: Pathophysiology, pathogenetic targets and clinical issues. Mol Aspects Med 2019;65:37-55.

30. Bataller R, Brenner DA, et al. Liver fibrosis. J Clin Invest 2005;115:209-18.

31. Hancox SH, Smith BC, et al. Liver disease as a cause of thrombocytopenia. QJM 2013;106:425-31.

32. Peck-Radosavljevic $M$. Thrombocytopenia in chronic liver disease. Liver Int 2017;37:778-93.

33. Sproston NR, Ashworth JJ, et al. Role of C-Reactive 
Protein at Sites of Inflammation and Infection. Front Immunol 2018;9:754.

34. Yoneda M, Mawatari H, Fujita K, et al. High-sensitivity C-reactive protein is an independent clinical feature of nonalcoholic steatohepatitis (NASH) and also of the severity of fibrosis in NASH. J Gastroenterol 2007;42:573-82.

35. Di Martino V, Coutris C, Cervoni JP, et al. Prognostic value of C-reactive protein levels in patients with cirrhosis. Liver Transpl 2015;21:753-60.

36. Cervoni JP, Amorós À, Bañares R, et al. Prognostic value of C-reactive protein in cirrhosis: external validation from the CANONIC cohort. Eur J Gastroenterol Hepatol 2016;28:1028-34.

(English Language Editor: B. Draper)
Cite this article as: Zeng M, Chen Y, Zhao B. Higher liver stiffness in patients with chronic congestive heart failure: data from NHANES with liver ultrasound transient elastography. Ann Palliat Med 2021;10(6):6859-6866. doi: 10.21037/apm-211362 